\title{
Exploring the Impulsion and Vibration Effects of Tactile Patterns
}

\author{
Muhammad Tahir \\ TELECOM ParisTech, LTCI CNRS, \\ Paris, France \\ tahir@telecom-parishtech.fr
}

\author{
Gilles Bailly \\ LIG, University of Grenoble 1, \\ Grenoble, France \\ gilles.bailly@telecom- \\ paristech.fr
}

\author{
Eric Lecolinet \\ TELECOM ParisTech, LTCI CNRS, \\ Paris, France \\ eric.lecolinet@telecom- \\ parishtech.fr
}

\begin{abstract}
This paper explores the impulsion and vibration properties of different tactile patterns. These properties describe the unique states of the pins for example raised or lowered. The tactile feedback is provided by Braille pins on a finger and is in the form of different patterns chosen for the experiments. We tested these features on the Braille display of dimension $4 \times 2$ ( 8 pins). The experiments were conducted to determine the selection time and error rate of impulsion and vibration characteristics. We report preliminary results of informal tests. We also discuss the related issues and propose possible future directions.
\end{abstract}

\section{Categories and Subject Descriptors}

H5.2. [Information interfaces and presentation]: User Interfaces, Haptic I/O.

\section{Keywords}

Tactile feedback, Braille cell, tactile patterns, impulsion, vibration, selection

\section{INTRODUCTION}

A Braille cell is normally intended for the visually impaired people. Such users move their finger on the Braille display and translate (read) the data. With a sufficient amount of training the user usually grasp the technique. Moreover, there exist situations where sighted users need to detect these patterns nonvisually for example talking to someone on phone, controlling a multimedia equipment (iPod, DVD Player for example) in a dimmed light room or using mobile phone in a meeting room [7] etc. This shows that the use of Braille cell for sighted people is equally difficult task if not more.

This problem gets multifold when the Braille cell dimensions are changed and user needs not move her/his fingers on large spatial area. In this situation the tactile pins continuously reveal the patterns (to be translated) on the fixed area of the cell. Scaling the problem to lower level, sometimes the users want to extract this specific information in less time. This problem has also been observed by [9]. The authors tested different sets of tactile patterns using one cell (dimension $4 \times 4$ ) of VTPlayer mouse (http://www.virtouch2.com) and reported selection time of more than one second per tactile pattern in every set.

(C) The Author 2008.

Published by the British Computer Society

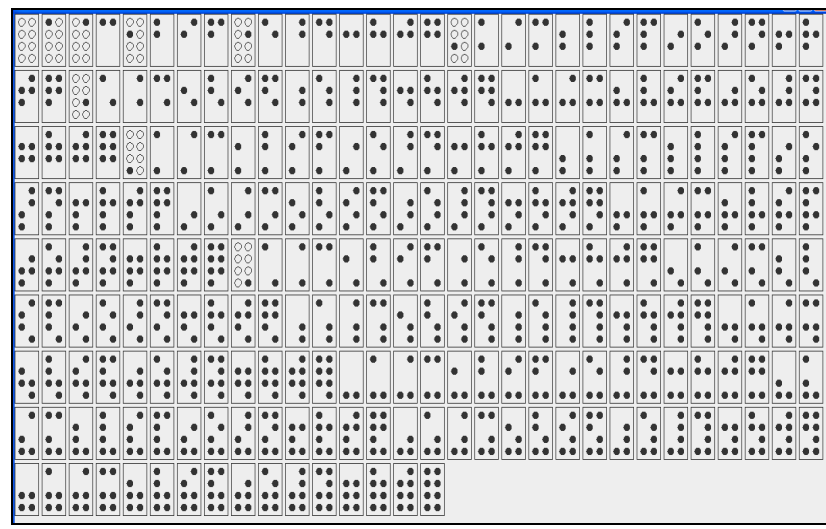

Figure 1: The possible 256 permutations of the $4 \times 2$ Braille cell

Our study enhances the work of [9] and explores the different dimensions of tactile patterns. However we test our experiment with somewhat different constraints. We use one $4 \times 2$ Braille cell (Metec ${ }^{\mathrm{TM}}$ : http://www.metec-ag.de/, 4 pins in one column $\mathrm{x}$ 2 pins in each row) for the tests as shown in figure 2 (left). We perform the experiments to determine the efficiency of the users for distinguishing tactile patterns. The objective is to determine the capacity of non-experts sighted users to recognize and memorize such tactile signals [12] based on their impulsive and vibration properties.

One important aspect of using customized Braille cells $\left(\mathrm{Metec}^{\mathrm{TM}}\right)$ is that they are refreshable i.e. while connected with the computer; the desired pins can be raised or lowered by applying different signals. This provides much flexibility in performing tests by applying variety of conditions and constraints. Moreover such devices are common, less expensive and allow decoding $2^{\mathrm{n}}$ possible permutations ( $\mathrm{n}$ is the number of pins on that customized tactile device).

The next section describes the previous work in this field. We then emphasize on the selection and the characteristics of different tactile patterns. Further, we present preliminary results obtained from the tests. Finally, we discuss the results and conclude our work.

\section{RELATED WORK}

A Braille cell is made up of six pins arranged in the form of two columns. Each column contains three pins. Many variants of such Braille system exist in which the pins can be raised or lowered depending upon the type of information one wants to read. The combination of different pins generates a pattern. The total number of patterns depends upon the number of pins used in that Braille cell. For example for 8 pins, 256 different possible patterns are possible (figure 1). The patterns can be used to behave differently. This behavior may be static or 
dynamic or both. For example one or more pins may remain static (in upward positions) or may move for certain duration of time. The information obtained from these tactile patterns can be used by visually impaired people [1] and as well as for sighted people [5].

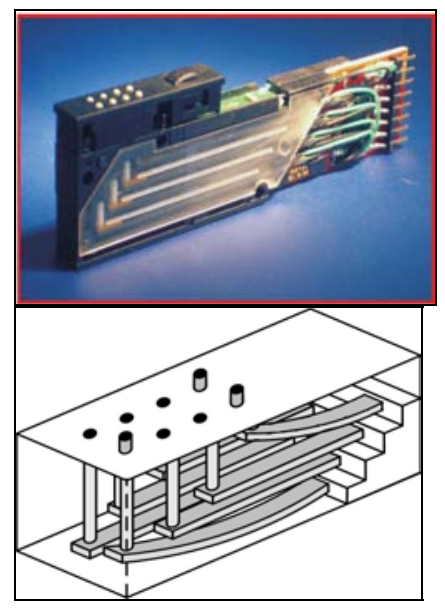

Figure 2: Top: A typical Metec ${ }^{\mathrm{TM}}$ Braille cell used (dimension: 4x2), Bottom: the Braille cell with tactile actuators

Three different tactile devices (TactiBall, TactiPen and TactiTab) have been proposed [5] which provide tactile feedback in different scenarios. In each device a $4 \times 2$ Braille cell is integrated and can simulate the functionality of the Trackball, PDA stylus and a Wacom-Pen. These devices provide the information difficult to be displayed on the small sized handheld screens. The tactile feedback is provided when the primary focus of the user is not on the Braille cell.

Ubi-Pen [4] is a tactile device which recognizes the Braille numbers from 0 to 9 . It integrates the customized Braille cell consisting of 9 pins $(3 \times 3)$. Tactile feedback is obtained by the textural representation of an image. When the Ubi-Pen is placed on the image, the gray scale portion of the image under the pen nib is divided into 9 equal squares. The textural intensity of each square causes the tactile pins to be raised up or lowered down. Results indicate that it improves the click sensation feedback. Moreover users were able to easily discriminate the tactile patterns with an average percentage of approximately $80 \%$.

VTPlayer mouse is tactile mouse in which two $4 \times 4$ (16 pins) sets of Braille cells are placed on the left and right click buttons. It has been used [9] to compare static and dynamic tactile patterns. The authors confirmed that the static patterns are efficient and can be easily detected. This study is in conformance with the solutions proposed by [1]. The task is to navigate in the virtual maze with cursor using dominant hand and receiving the tactile information on the non-dominant hand. The raised pins represent the wall and lowered pins indicate the safe passage (along the direction of motion of the cursor). Results indicate that the users performed faster in the condition when pins stayed static. Our study is rather based on different conditions: first we use $4 \times 2$ Braille cell contrary to the above experiments. Second, we investigated the different characteristics of the tactile patterns for example the impulsion and the dynamicity, which are actually the variations of static and dynamic patterns respectively.

\section{TACTILE PATTERNS}

The tactile Braille cell used is the combination of different piezoelectric actuators or bimorphs (figure 2, right). These actuators are thus used to move the 8 pins in a specific order. Each pin can have two different states: raised or lowered (similar to 1 and 0 bits of information for each state). Different pins in different states can represent different types of information. For example all pins in raised or lowered states represent information say info1, while some pins in raised or lowered or combined state represent different information say info2. The state of the pins can be controlled by the signal applied on them via software application.

\subsection{The Choice of Tactile Patterns}

A tactile pattern may contain zero or more pins. Consider the example of figure 1 which is a large set of patterns and difficult to test and evaluate in a limited time. Thus it becomes necessary to select and test some of these tactile patterns.

We used the combination of two techniques to select a small set of patterns for our experiments. The first technique uses the approach presented in [8] and the second technique uses heuristic method. The first technique introduces the notion of distance (codes) between different tactile patterns in order to discriminate them. These codes are determined by considering the raised or lowered states of the pins and their positions on the Braille cell.

In the heuristic approach we first filter out the patterns with lower code values i.e. the patterns (left and lower regions of the figure 1) with higher number of raised pins in close proximity. Moreover, some patterns (middle and right regions of the figure 1) were discarded because their irregular shapes create higher cognitive load for their detection and recognition.

We randomly tested some of these selected patterns but found them inefficient due to the higher selection time and the memorization problem. Moreover in accordance with the results of [9], on the first site, we have tested almost 25 patterns. More tests on this set resulted even less number of patterns (figure 3 ) that can easily be recognized. However the above mentioned selection procedures can still be refined which may further introduce the inclusion or exclusion of different patterns.

To favor memorization we can categorize the tactile patterns of figure 3 as: the patterns 0 and 1 represent one and all pins in raised states thus easy to remember the cutaneous impact on the finger. The patterns 2 and 3 represent four pins each in the center and on the top and bottom rows. The remaining patterns have 2 pins thus covering the upper and lower corners and creating a sequence of top-left, top-right, bottom-left, bottomright.

\subsection{Tactile Pattern Types}

Basically there are two types of tactile patterns: static and dynamic. Static patterns are the patterns which remain on the raised state until detected or for certain duration of time. The dynamic patterns are the patterns which move on the Braille cell, the number of times as specified by the computer system application. Thus the vibrating patterns are the simulation of animated pins. Pietrzak [9] and Crossan [1] have confirmed that the static patterns can be easily detected than that of dynamic ones. In this study we are using the variation of static and dynamic patterns which are named as impulsion and vibration (oscillation) respectively.

An impulse pattern is the pattern which is displayed suddenly and once. The duration during which it remains raised is 
controlled by applying short delay up time and longer delay down time. This sudden impact on the finger skin with frequency of $3 \mathrm{~Hz}$ [4] improves the percent of correct discrimination of tactile pattern on human skin [3].

The vibrating pattern is raised up for $n$ number of times during a specified time interval. The value of $n$ is dependent on this interval which is defined at the start of the experiment. We have reserved this time as one second. This is the time during which a user can easily feel the certain sequences of one vibrating pattern enough number of times in order to detect and distinguish it from other patterns. This result is taken from [10] which resulted that settling time response of the beam tip of the piezoelectric bimorph is around 150 to 170 milliseconds. To be on the safe side we used the delay up value of $200 \mathrm{~ms}$ for each sequence of vibrating patterns.

\begin{tabular}{|c|c|c|c|c|c|c|c|}
\hline 0 & 1 & 2 & 3 & 4 & 5 & 6 & 7 \\
\hline - 0 & • & 00 & $\bullet \bullet$ & - 0 & $\circ \bullet$ & 00 & $\circ 0$ \\
\hline 00 & $\bullet$ & $\bullet \bullet$ & 00 & - 0 & $\circ \bullet$ & 00 & O \\
\hline 00 & $\bullet$ & $\bullet \bullet$ & 00 & 00 & 00 & - 0 & 0 \\
\hline 00 & $\bullet$ & 00 & $\bullet \bullet$ & 00 & 00 & - 0 & 0 \\
\hline
\end{tabular}

Figure 3: Each tactile pattern represents different pins used in experiments. Raised pins are represented as black dots.

\section{EXPERIMENT}

The goal of the experiment was to detect and distinguish different tactile patterns based on their impulsive and vibration characteristics. This was accomplished by selecting the target from a set of different tactile patterns.

All patterns including stimulus were displayed on a laptop screen. This helped the subjects to compare the patterns (under finger) with the patterns on the screen. The experiment was implemented with $\mathrm{C}++$ and the ubit toolkit [6]. We used HP Compaq nw8240 system and 1 Braille cell as tactile feedback. The dimension of the Braille cell was 6.42 (width) x 16.7 (height) $\mathrm{x} 74.60$ (depth) $\mathrm{mm}$ with pin spacing of $2.45 \mathrm{~mm}$. The Braille cell was integrated inside a box displaying the pins on the surface. The subject can place her/his hand on the box to avoid fatigue. Eight different tactile patterns (figure 3) were chosen for the experiments.

We have used with-in subject experimental design because it allows performing the test equally and with all conditions. The subjects performed the impulsion test first and then the vibration tests. The test procedures were explained before the actual experiments. Each subject performed at least two test blocks as training. The goal was to quickly and accurately select the target pattern as soon as it is felt under the finger.

At the start of the trial, the stimulus appeared on the screen by pressing the spacebar. The patterns then started appearing on the Braille cell one after another with a time interval of one second. The subject feels different patterns moving under the finger. As soon as the subject detects the target pattern s/he presses again the spacebar to select it. The selected pattern along with different information is logged and the experiment continues for the next block. Visual feedback (correct / incorrect) as text was provided on the screen. An error was equally logged if the subject failed to detect the target. Each pattern including target appeared once and at random positions in each block. Each pattern in all blocks appeared equal number of times.

Our hypotheses were: 1) the average target selection time would be less in vibration (set 4) than the average selection time of impulsion 2) the vibration test will produce lower number of errors because each subject has enough time to detect and judge the target pattern 3 ) the increase in impulsion delay up time may decrease the selection time.

We have further subdivided the set given in the figure 3 . For the impulsion test, we used 4 patterns (patterns 0 to 3 ). For vibration test, we used 2 sets: the vibration set 4 (patterns 0 to 3 ) and vibration set 8 (all patterns). The reason of performing tests with vibrating set 8 was to observe the shift in learning and remembrance behavior of patterns from set 4 to set 8 . Counting the number of errors reflected this behavior.

\section{RESULTS AND DISCUSSION}

We have tested the impulsion feature with customized values of delay up time (values in the range of $100 \mathrm{~ms}$ to $250 \mathrm{~ms}$ with the difference of 25ms). These values indicate different impulsion effects because the increase in delay up time causes the pins to stay longer and may be recognized efficiently.

The results indicate that patterns 0 and 1 can be quickly detected $(595 \mathrm{~ms})$ at the delay up value of $150 \mathrm{~ms}$. While in case of pattern 2 and 3 this value is $130 \mathrm{~ms}$ and $175 \mathrm{~ms}$ respectively. This may be the case because patterns 0 and 1 have simple arrangement of pins. Moreover, the result indicates that the increase in delay up time has no effect on the decrease in selection time, thus invaliding our third hypothesis.

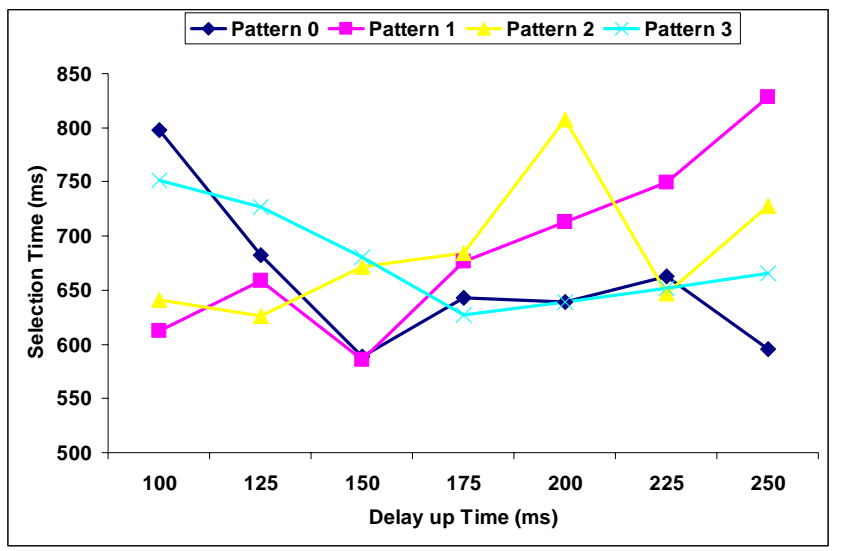

Figure 4: Average selection time for an impulsion set of 4 patterns with different values of delay up time.

The result of vibrating sets (Figure 5) indicates that the selection time is in the range of $615 \mathrm{~ms}$ to $670 \mathrm{~ms}$. By increasing the number of patterns twofold, the number of errors gets almost doubled so as the selection time (Figure 6). This clearly indicates that in this situation it becomes gradually difficult for the users to remember different patterns. The results in both cases indicate that the average selection time for impulsion is $680 \mathrm{~ms}$ and for vibration it is $615 \mathrm{~ms}$. This conforms to our first hypothesis.

In vibration test we come across an interesting result which indicates that the increase in the number of errors was due to the less time left between the appearances of two consecutive patterns. The original delay up time was $200 \mathrm{~ms}$. Add some time for the pins to drop and stay lowered say 75 to $100 \mathrm{~ms}$. Thus during threshold time user felt the target at least three times, but at the end there was not enough time left to distinguish the current pattern from the new pattern. 


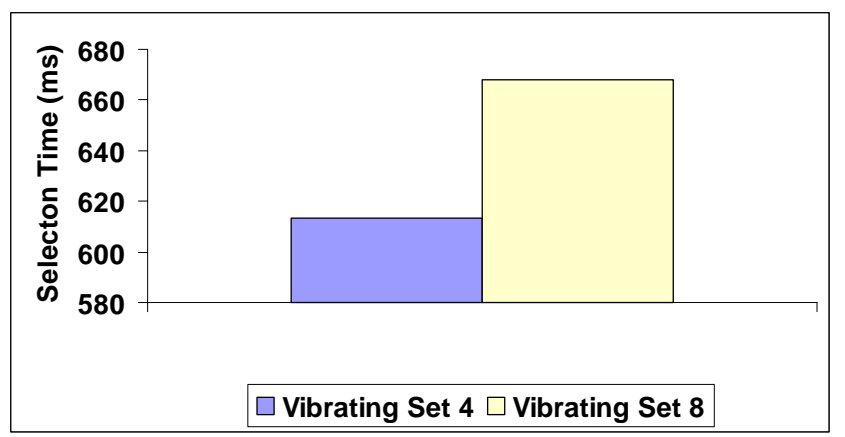

Figure 5: Average selection time for vibrating set of 4 and 8 patterns.

Although the number of errors increased in increasing the number of tactile patterns but still it also depends on the type of pattern selected. The higher the distance value (and simple shape) higher are the chances for the tactile pattern to get recognized.

In vibrating test, it has been observed that the subjects performed the passive touch (hold the finger over the pattern). While in impulsion, sometimes the users performed the active touch (explored the pattern by moving the finger on it). This may be due to the refreshable presentation of the Braille used in our experiment. This is not the same case as of original Braille where the users perform active touch most of the time.

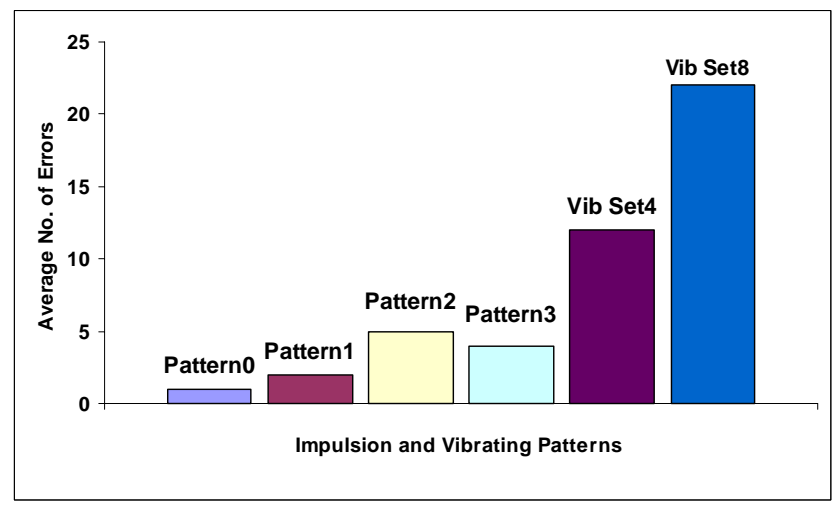

Figure 6: Average number of errors for impulsion set 4 (range of different frequencies) and for vibrating sets 4 and 8.

With the first contact of vibrating pattern, if the user is not sure about the target, s/he must have to wait for the second cycle (after 200ms) to reconfirm it. This results in the increase in the selection time but reduces the error rate.

The discussion after the tests revealed that some users tried to remember the impact of certain pins at the specified position of the finger (especially in impulsion case). This is almost the same situation observed by [11] which shows that the remembrance of items in the list depends upon the frequency of access and on items location on the screen. A similar situation [2] provides users the ability to memorize and recognize unique tactile feedback for a password authentication system.

\section{CONCLUSION AND FUTURE WORK}

In this paper we presented the impulsion and vibration characteristics of the tactile patterns. Results indicate that, though the selection time with less than one second, both features behave differently under different conditions: for example delay up time, active or passive touch and the number of errors.
As future work, we plan to perform precise evaluation of these characteristics with different constraints. We also like to refine the techniques for selecting optimal tactile patterns in the set of $2^{8}$ patterns. We also plan to perform these tests on the $4 \times 4$ Braille cell with an option of choosing variety of tactile patterns.

\section{ACKNOWLEDGMENTS}

We thank Anne Roudaut, Sylvain Malacria and Gérard Mouret for their valuable suggestions. We also thank HEC \& CIIT Islamabad for their financial and moral support.

\section{REFERENCES}

1. Crossan A, Brewster S. Two handed navigation in a haptic virtual environment. In Proceedings CHI 06, p. 676-81.

2. Kuber R., Yu W., Authentication using tactile feedback, Interactive Experiences, HCI 2006, London, UK, p. 11-15

3. Kyung, K., Ahn, M., Kwon, D., and Srinivasan, M. A. Perceptual and Biomechanical Frequency Response of Human Skin Implication for Design of Tactile Displays. In Proceedings of Eurohaptics 2005. p. 96-101

4. Kyung, K. and Park, J. 2007. Ubi-Pen: Development of a Compact Tactile Display Module and Its Application to a Haptic Stylus. In Proceedings of Eurohaptics 2007, p.109114.

5. Lecolinet E., and Mouret G. tactiball, tactipen, tactitab, ou comment toucher du doigt, les données de son ordinateur. In Proceedings of IHM 05, p. 227-30.

6. Lecolinet E. A molecular architecture for creating advanced GUIs. In Proceedings of UIST 03, p. 135-44.

7. Luk J., Pasquero J., Little S., Maclean K., Lévesque V. and Hayward V. A role for Haptic in Mobile Interaction: Initial Design Using a Handheld Tactile Display Prototype. In Proceedings of CHI 06, p. 171-80.

8. Mandic P. Danilo., Havey Richard and Kolonic H. Djemal. On the choice of tactile code. In the proceedings of IEEE Multimedia and Expo, 2000, p. 195-198.

9. Pietrzak T., Pecci I., Martin B. Static and dynamic tactile directional cues experiments with VTPlayer mouse. In Eruohaptics 06, Paris, France.

10. Smithmaitrie, P., Kanjantoe, J., and Tandayya, P. Touching force response of the piezoelectric Braille cell. In Proceedings of i-CREATe '07, p. 174-178.

11. Teevan, J. How people recall search result lists. In Proceedings of CHI'06, p. 1415-1420

12. Ziat M., Gapenne O., Lenay C. and Stewart J. Acuité perceptive via une interface pseudo-haptique. IHM 04, p.263-266 